\title{
A DOUBLE WEIGHT EXTRAPOLATION THEOREM
}

\author{
C. J. NEUGEBAUER
}

\begin{abstract}
If an operator is of weak type $\left(p_{0}, p_{0}\right)$ with weights $(u, v)$ for every
\end{abstract} $(u, v) \in A_{p_{0}}$, then the same holds for $1<p<p_{0}$.

1. A nonnegative function $u$ on $\mathbf{R}^{n}$ is said to be in $A_{p}$ iff $\left(\int_{Q} u\right) \cdot\left(\int_{Q} u^{1-p^{\prime}}\right)^{p-1} \leqslant$ $c|Q|^{p}$, and a pair of nonnegative functions $(u, v)$ is in $A_{p}$ iff $\left(\int_{Q} u\right)\left(\int_{Q} v^{1-p^{\prime}}\right)^{p-1} \leqslant$ $c|Q|^{p}$. The smallest $c$ for which these inequalities hold for all cubes $Q \subset \mathbf{R}^{n}$ will be referred to as the $A_{p}$-constant of $u,(u, v)$, respectively. These classes were introduced by Muckenhoupt [3] and are important in the study of weighted norm inequalities for the Hardy-Littlewood maximal operator

$$
M f(x)=\sup _{x \in Q} \frac{1}{|Q|} \int_{Q}|f|,
$$

the Hilbert transform, and many others. In [1] we find the following remarkable theorem.

THEOREM 1. Let $T$ be a sublinear operator, $1 \leqslant p_{0}<\infty$, and

$$
u\{x:|T f(x)|>y\} \leqslant \frac{B}{y^{p_{0}}} \int|f|^{p_{0}} u \text { for every } u \in A_{p_{0}},
$$

where $B$ depends only on the $A_{p_{0}}$-constant of $u$. Then, if $1<p<\infty$ and $u \in A_{p}$,

$$
u\{x:|T f(x)|>y\} \leqslant \frac{C}{y^{p}} \int|f|^{p} u,
$$

where $C$ depends only on the $A_{p}$-constant of $u$.

This theorem remains true if all weak type inequalities are replaced by strong type inequalities, and in this setting was first proved by Rubio de Francia [5]. The method of the proof of Theorem 1 given by Garcia-Cuerva in [1] is different and, as we shall see, can be modified to obtain a double weight $(u, v)$ version of Theorem 1 .

2. The results which we obtain are best stated in the terminology of $L(p, q, \lambda)$ spaces [2]. If $\lambda$ is a Borel measure on $\mathbf{R}^{n}$ and, for $f: \mathbf{R}^{n} \rightarrow \mathbf{R}$,

$$
f_{\lambda}^{*}(t)=\inf \{y: \lambda\{|f|>y\} \leqslant t\}
$$

Received by the editors May 15, 1984.

1980 Mathematics Subject Classification. Primary 42B25. 
the nonincreasing rearrangement of $f$ with respect to the measure $\lambda$, then we write

$$
\|f\|_{p, q ; \lambda}=\left\{\int_{0}^{\infty}\left[t^{1 / p} f_{\lambda}^{*}(t)\right]^{q} \frac{d t}{t}\right\}^{1 / q}, \quad \text { if } 1 \leqslant p, q<\infty,
$$

and $\|f\|_{p, \infty ; \lambda}=\sup _{t>0} t^{1 / p} f_{\lambda}^{*}(t), 1 \leqslant p<\infty$. If $d \lambda=u d x$, we write $\|f\|_{p, q: u}=$ $\|f\|_{p, q ; \lambda}$. The weak type inequalities in Theorem 1 can then be written as $\|T f\|_{p, \infty: u}$ $\leqslant C\|f\|_{p, p ; v} \equiv C\|f\|_{p, v}$.

The method in [1] carries immediately over to prove the following

THEOREM 2. Let $T$ be a sublinear operator, $1 \leqslant p_{0}<\infty$, and $\|T f\|_{p_{0}, \infty: u} \leqslant B\|f\|_{p_{0}, v}$, for every $(u, v) \in A_{p_{0}}$, where $B$ depends only on the $A_{p_{0}}$-constant of $(u, v)$. Let $1<p<\infty$, and let $(u, v) \in A_{p}$ such that $\|M f\|_{p^{\prime}, v^{1-p^{\prime}}} \leqslant c\|f\|_{p^{\prime}, u^{1-p^{\prime}}}$ and $\|M f\|_{p, u} \leqslant$ $c\|f\|_{p, v}$. Then $\|T f\|_{p, \infty ; u} \leqslant C\|f\|_{p, v^{\prime}}$ where $C$ depends only on the $A_{p}$-constant of $(u, v)$.

The proof is exactly the same as the one given in [1] except now $G=$ $\left\{M .\left(g^{1 / t} u\right) v^{-1}\right\}^{t}$. The strong type norm inequalities on the maximal function make Lemmas 1,2 of [1] valid statements, i.e.,

Lemma 1. Let $(u, v) \in A_{p}$ for some $1<p<\infty$, and for $0<t \leqslant 1$ and $g \geqslant 0$, let $G=\left\{M\left(g^{1 / t} u\right) v^{-1}\right\}^{t}$.

(i) If $p_{0}=p-t p / p^{\prime}$, then $(g u, G v) \in A_{p_{0}}$ with $A_{p_{0}}$-constant no larger than the $A_{p}$-constant of $(u, v)$ raised to the $1-t$ power.

(ii) If $\|M f\|_{p^{\prime}, v^{1-p^{\prime}}} \leqslant C\|f\|_{p^{\prime}, u^{1-p^{\prime}}, \text { then }}\|G\|_{p^{\prime} / t, v} \leqslant C\|g\|_{p^{\prime} / t, u^{\prime}}$.

3. It is well known that $(u, v) \in A_{p}$ if and only if $\left(v^{1-p^{\prime}}, u^{1-p^{\prime}}\right) \in A_{p^{\prime}}$, and that these are equivalent with $\|M f\|_{p, \infty ; u} \leqslant B\|f\|_{p, v}$. However, strong type inequalities for $M$ need not hold (see [4]), and thus the strong type inequalities for $M$ in the hypothesis of Theorem 2 are unnatural. We will prove the following

THEOREM 3. Let $1<p_{0}<\infty$, and let $T$ be a sublinear operator such that $\|T f\|_{p_{0}, \infty ; u}$ $\leqslant B\|f\|_{p_{0}, v}$ for every $(u, v) \in A_{p_{0}}$, where $B$ depends only upon the $A_{p_{0}}$-constant of $(u, v)$. If $1<p<p_{0}$ and $(u, v) \in A_{p}$, then $\|T f\|_{p, \infty: u} \leqslant C\|f\|_{p, v}$, where $C$ depends only upon the $A_{p}$-constant of $(u, v)$.

The proof is based on the following

Lemma 2. Let $1<p<p_{0}<\infty,(u, v) \in A_{p}$, and $g \geqslant 0$ in $L^{p /\left(p_{0}-p\right)}(v)$. Then there exists a function $G \geqslant 0$ such that

$$
u(G>y) \leqslant \frac{c}{y^{p /\left(p_{0}-p\right)}} \int g^{p /\left(p_{0}-p\right)} v, \text { and }
$$

(ii) $\left(G^{-1} u, g^{-1} v\right) \in A_{p_{0}}$, where the constants involved depend only on the $A_{p}$-constant of $(u, v)$.

Proof. We note that $p_{0}^{\prime}<p^{\prime}$ and $\left(v^{1-p^{\prime}}, u^{1-p^{\prime}}\right) \in A_{p^{\prime}}$. We set

$$
t=\left(p^{\prime}-p_{0}^{\prime}\right) /\left(p^{\prime}-1\right)
$$

so that $p_{0}^{\prime}=p^{\prime}-t p^{\prime} / p$ and $(p / t)^{\prime}=p^{\prime} / p_{0}^{\prime}$. We define $h$ by the relation

$$
g^{p /\left(p_{0}-p\right)} v=h^{p\left(p_{0}-1\right) /\left(p_{0}-p\right)} v^{1-p^{\prime}},
$$


and we set (as in Lemma 1)

$$
H=\left\{M\left(h^{1 / t} v^{1-p^{\prime}}\right) u^{p^{\prime}-1}\right\}^{t} .
$$

Since $(u, v) \in A_{p}$, we get

$$
u\left\{M\left(h^{1 / t} v^{1-p^{\prime}}\right)>y^{1 / t}\right\} \leqslant \frac{c}{y^{p / t}} \int h^{p / t} v^{1-p^{\prime}},
$$

and hence

$$
u\left\{H u^{t\left(1-p^{\prime}\right)}>y\right\} \leqslant \frac{c}{y^{p / t}} \int h^{p / t} v^{1-p^{\prime}} .
$$

We now let $G=H^{p_{0}-1} u^{-\left(p_{0}-p\right) /(p-1)}$, and using (1) we check that

$$
u\{G>y\} \leqslant \frac{c}{y^{p /\left(p_{0}-p\right)}} \int g^{p /\left(p_{0}-p\right)} v .
$$

Since, by Lemma $1,\left(h v^{1-p^{\prime}}, H u^{1-p^{\prime}}\right) \in A_{p_{0}^{\prime}}$, we see that

$$
\left(H^{1-p_{0}} u^{\left(1-p^{\prime}\right)\left(1-p_{0}\right)}, h^{1-p_{0}} v^{\left(1-p^{\prime}\right)\left(1-p_{0}\right)}\right) \in A_{p_{0}} .
$$

The proof is completed by noting that $G^{-1} u=H^{1-p_{0}} u^{\left(p_{0}-1\right)\left(p^{\prime}-1\right)}$, and $g^{-1} v=$ $h^{1-p_{0}} v^{\left(1-p^{\prime}\right)\left(1-p_{0}\right)}$.

Proof of Theorem 3. Let $E_{s}=\{x:|T f(x)|>s\}$. Note that $\|f\|_{p, v}^{p_{0}}=$ $\left\||f|^{p_{0}}\right\|_{p / p_{0}, v}=\int|f|^{p_{0}} g^{-1} v$ for some $\int g^{p /\left(p_{0}-p\right)} v=1$. If $r=p / p_{0}$, then $s^{p_{0}} u\left(E_{s}\right)^{p_{0} / p}=s^{p_{0}}\left(\int \chi_{E_{s}} u\right)^{1 / r}=s^{p_{0}}\left\{\int \chi_{E_{s}} G \cdot G^{-1} u\right\}^{1 / r}$, where $G$ is as in Lemma 2. By Hölder's inequality we obtain $s^{p_{0}} u\left(E_{s}\right)^{p_{0} / p} \leqslant s^{p_{0}}\left\|\chi_{E_{s}}\right\|_{\sigma, 1 ; G^{-1} u}^{1 / r} \cdot\|G\|_{\sigma^{\prime}, \infty ; G^{-1} u}^{1 / r}$, where $\sigma=1 / r$.

We first estimate $\|G\|_{\sigma^{\prime}, \infty: G^{-1} u}$. The distribution function of $G$ with respect to the measure $G^{-1} u d x$ is

$$
G^{-1} u\{G(x)>y\}=\int_{\{G(x)>y\}} G^{-1} u \leqslant \frac{1}{y} \int_{\{G(x)>y\}} u \leqslant \frac{c}{y^{p_{0} /\left(p_{0}-p\right)}}
$$

by (i) of Lemma 2. Hence

$$
(G)_{G^{-1} u}^{*}(t) \leqslant \frac{c}{t^{\left(p_{0}-p\right) / p_{0}}}=\frac{c}{t^{1 / \sigma^{\prime}}},
$$

and so

$$
\|G\|_{\sigma^{\prime}, \infty: G^{-1} u}=\sup _{t>0}\left\{t^{1 / \sigma^{\prime}}(G)_{G^{-1} u}^{*}(t)\right\} \leqslant c .
$$

For the estimation of

$$
\left\|\chi_{E_{s}}\right\|_{\sigma, 1: G^{-1} u}=\int_{0}^{\infty} t^{1 / \sigma}\left(\chi_{E_{s}}\right)_{G^{-1} u}^{*}(t) \frac{d t}{t},
$$

we note that

$$
\begin{aligned}
G^{-1} u\left\{\chi_{E_{s}}>y\right\} & =G^{-1} u\left(E_{s}\right) \cdot \chi_{[0,1]}(y) \\
& \leqslant \frac{c}{s^{p_{0}}}\|f\|_{p, v^{\prime}}^{p_{0}} \cdot \chi_{[0,1]}(y) .
\end{aligned}
$$


From this we obtain that

$$
\left(\chi_{E_{s}}\right)_{G^{-1} u}^{*}(t) \leqslant \chi_{[0, R]}(t), \quad \text { where } R=\frac{c}{s^{p_{0}}}\|f\|_{p, v}^{p_{0}},
$$

and, consequently,

$$
\left\|\chi_{E_{s}}\right\|_{\sigma, 1 ; G^{-1} u} \leqslant \int_{0}^{R} t^{1 / \sigma-1} d t=c R^{1 / \sigma}=c\left(\frac{1}{s^{p_{0}}}\|f\|_{p, v}^{p_{0}}\right)^{r} .
$$

This implies that

$$
s^{p_{0}} u\left(E_{s}\right)^{p_{0} / p} \leqslant c s^{p_{0}} \cdot \frac{1}{s^{p_{0}}}\|f\|_{p, v}^{p_{0}} \quad \text { or } \quad s u\left(E_{s}\right)^{1 / p} \leqslant c\|f\|_{p, v},
$$

which was to be proved.

4. It is not known to the writer whether Theorem 3 is true if $p>p_{0}$. In order to formulate a substitute result for $p>p_{0}$ we rewrite

$$
\begin{aligned}
\int|f|^{p} v & =\int|f|^{p} v^{p^{\prime}} v^{1-p^{\prime}}=\int\left(|f| v^{p^{\prime}-1}\right)^{p} v^{1-p^{\prime}} \\
& =\int_{0}^{\infty}\left(f v^{p^{\prime}-1}\right)_{v^{1-p^{\prime}}}^{* p}(t) d t=\left\|f v^{p^{\prime}-1}\right\|_{p, p: v^{1-p^{\prime}}}^{p}
\end{aligned}
$$

THEOREM 4. Let $1<p_{0}<p<\infty$, and let $T$ be a sublinear operator so that, for every $(u, v) \in A_{p_{0}}$

$$
\|T f\|_{p_{0}, \infty, u} \leqslant c\|f\|_{p_{0} v}=c\left\|f v^{p_{0}^{\prime}-1}\right\|_{p_{0}, p_{0} ; v^{1-p_{o}^{\prime}}},
$$

where $c$ only depends on the $A_{p_{0}}$-constant of $(u, v)$. Then, if $(u, v) \in A_{p}$,

$$
\|T f\|_{p, \infty, u} \leqslant c\left\|f v^{p^{\prime}-1}\right\|_{p, p_{0} ; v^{1-p^{\prime}}}
$$

where $c$ only depends upon the $A_{p}$-constant of $(u, v)$.

REMARK. Since $\left\|f v^{p^{\prime}-1}\right\|_{p, p_{0} ; v^{1-p^{\prime}}} \geqslant\left\|f v^{p^{\prime-1}}\right\|_{p, p ; v^{1-p^{\prime}}}=\|f\|_{p, v}$ (see [2]), the conclusion of Theorem 4 is not as strong as the corresponding weak type inequality.

The proof is based on a slight reformulation of Lemma 1, which we state as

LemMA 3. Let $1<p_{0}<p<\infty,(u, v) \in A_{p}$, and let $t=\left(p-p_{0}\right) /(p-1)$. If $g \geqslant 0$ is in $L^{p /\left(p-p_{0}\right)}(u)$, and $G=\left\{M\left(g^{1 / t} u\right) v^{-1}\right\}^{t}$, then

$$
v^{1-p^{\prime}}\left\{M\left(g^{1 / t} u\right)^{t}>y\right\} \leqslant \frac{c}{y^{p /\left(p-p_{0}\right)}} \int g^{p /\left(p-p_{0}\right)} u, \quad \text { and }
$$

(ii) $(g u, G v) \in A_{p_{0}}$.

The constants in (i) and (ii) depend only on the $A_{p}$-constant of $(u, v)$.

Proof. Since $\left(v^{1-p^{\prime}}, u^{1-p^{\prime}}\right) \in A_{p^{\prime}}$ with the same $A_{p^{\prime}}$-constant as the $A_{p}$-constant for $(u, v)$, we see that

$$
\int_{\left\{M\left(g^{1 / t} u\right)>y^{1 / t}\right\}} v^{1-p^{\prime}} \leqslant \frac{c}{y^{p^{\prime} / t}}\|g\|_{p^{\prime} / t, u}^{p^{\prime} / t}
$$

and this is (i). The proof of (ii) is exactly the same as in [1]. 
Proof of Theorem 4. Let $E_{s}=\{x:|T f(x)|>s\}$ and note that $s^{p_{0}} u\left(E_{s}\right)^{p_{0} / p}=$ $s^{p_{0}}\left\|\chi_{E_{s}}\right\|_{p / p_{0}, u}=s^{p_{0}} \int \chi_{E_{s}} g u$, for some $\|g\|_{\left(p / p_{0}\right)^{\prime}, u}=1$. By (ii) of Lemma 3,

$$
\begin{aligned}
s^{p_{0}} u\left(E_{s}\right)^{p_{0} / p} & \leqslant c \int|f|^{p_{0}} G v=c \int|f|^{p_{0}} v^{p^{\prime}-t} M\left(g^{1 / t} u\right)^{t} v^{1-p^{\prime}} \\
& \leqslant c\left\||f|^{p_{0}} v^{p^{\prime}-t}\right\|_{\left(p^{\prime} / t\right)^{\prime}, 1 ; v^{1-p^{\prime}}}\left\|M\left(g^{1 / t} u\right)^{t}\right\|_{p^{\prime} / t, \infty ; v^{1-p^{\prime}}} \\
& \leqslant c\left\|f v^{p^{\prime}-1}\right\|_{p, p_{0}: v^{1-p^{\prime}}}^{p_{0}}
\end{aligned}
$$

since $\left(p^{\prime} / t\right)^{\prime}=p / p_{0}$. This completes the proof.

\section{REFERENCES}

1. J. Garcia-Cuerva, An extrapolation theorem in the theory of $A_{p}$ weights, Proc. Amer. Math. Soc. 87 (1983), 422-426.

2. R. A. Hunt, On $L(p, q)$ spaces, Enseign. Math. (2) 12 (1966), 249-275.

3. B. Muckenhoupt, Weighted norm inequalities for the Hardy maximal function, Trans. Amer. Math. Soc. 165 (1972), 207-226.

4. B. Muckenhoupt and R. Wheeden, Two weight function norm inequalities for the Hardy-Littlewood maximal function and the Hilbert transform, Studia Math. 55 (1976), 279-294.

5. J. L. Rubio de Francia, Factorization and extrapolation of weights, Bull. Amer. Math. Soc. (N. S.) 7 (1982), 393-396.

Department of Mathematics, Purdue University, West Lafayette, Indiana 47907 